\title{
Capítulo 9 Calidad de la carne de las especies domésticas
}

\section{Chapter 9 Quality of meat from domestic species}

BAUTISTA-MARTINEZ, Yuridia ${ }^{1} \dagger^{*}$, CRUZ-TAMAYO, Alvar Alonzo ${ }^{2}$ y GRANADOS-RIVERA, Lorenzo Danilo ${ }^{3}$

${ }^{1}$ Universidad Autónoma de Tamaulipas. Facultad de Medicina Veterinaria y Zootecnia. Carretera Mante Km 5. C.P. 87000. Ciudad Victoria Tamaulipas, México.

${ }^{2}$ Universidad Autónoma de Campeche, Escuela Superior de Ciencias Agropecuarias, Calle 53 S/N, Col. Unidad, Esfuerzo y Trabajo No. 2. C.P. 24350, Escárcega, Campeche, México

${ }^{3}$ Instituto Nacional de Investigaciones Forestales, Agrícolas y Pecuarias, C.E. General Terán, Carretera Montemorelos-China, Nuevo León, México

ID $1^{\mathrm{er}}$ Autor: Yuridia, Bautista-Martinez / ORC ID: 0000-0003-1707-4877, CVU CONACYT ID: 383767

ID $1^{\text {er }}$ Coautor: Alvar Alonzo, Cruz-Tamayo / ORC ID: 0000-0002-5509-3430, CVU CONACYT ID: 83358

ID $2^{\text {do }}$ Coutor: Lorenzo Danilo, Granados-Rivera /ORC ID: 0000-0002-8502-4612, CVU CONACYT ID: 383776

DOI: $10.35429 /$ H.2020.2.158.174

Y. Bautista, A. Cruz y L. Granados

ybautista@docentes.uat.edu.mx

N. Niño, M. Valencia y M. García. (AA. VV.) Sustentabilidad, Turismo y Educación. Handbooks-TII-@ECORFAN-Mexico, Guerrero, 2020. 


\title{
Resumen
}

El objetivo principal de este capítulo es proporcionar al lector los conceptos básicos sobre la calidad de la carne de especies domésticas. Para lograr este objetivo se dividió el texto en tres partes, la primera parte trata de las generalidades de la carne donde se aborda la estructura del músculo y los procesos bioquímicos que ocurren para que el músculo se convierta en carne. La segunda parte contempla la calidad de la carne y su clasificación fisicoquímica, así como el efecto del estrés ante mortem en la calidad de esta. La parte final aborda el tema de la carne y su importancia nutrimental, donde se hace énfasis en que el consumo de carne por los humanos es esencial para su crecimiento y desarrollo, también se abarca el tema de las modificaciones que puede hacerse a la carne y los productos cárnicos para enriquecerlos y ser considerados alimentos funcionales. Finalmente, en este mismo apartado, se pone de manifiesto la importancia de la inocuidad alimentaria especialmente en el riesgo de contraer enfermedades por consumo de carne contaminada con bacterias patógenas.

\section{Composición química de la carne, Alimento funcional, Escherichia coli 0157:H7, Campylobacter jejuni}

\begin{abstract}
The main objective of this chapter is to provide the reader with basic concepts about the quality of meat from domestic species. To achieve this goal the text was divided into three parts, the first part deals with the generalities of the meat where the muscle structure is addressed and the biochemical processes that occur so that the muscle becomes flesh. The second part covers the quality of meat and its physicochemical classification, as well as the effect of ante-mortem stress on meat quality. His final part deals with the topic of meat and its nutritional importance, where it is emphasized that the consumption of meat by humans is essential for its growth and development, it also covers the issue of possible modifications to meat and meat products to enrich them and to be considered functional foods. Finally, this same paragraph highlights the importance of food safety, especially in terms of the risk of disease from eating meat contaminated with pathogenic bacteria.
\end{abstract}

\section{Chemical composition of meat, Functional food, Escherichia coli 0157:H7, Campylobacter jejuni}

\section{Introducción}

La carne se define como todo tejido animal apto para el consumo humano. En general, se compone de agua, proteínas, grasas, minerales y una pequeña proporción de carbohidratos. Desde el punto de vista nutricional la proteína es el componente más valioso. El contenido de humedad es el más variable, y está estrecha e inversamente relacionado con su contenido de grasa. En el cuerpo animal, aproximadamente el $65 \%$ de las proteínas provienen del músculo esquelético, $30 \%$ del tejido conectivo (colágeno, elastina) y $5 \%$ de proteínas sanguíneas, queratina en pelos y uñas. La composición química de los cortes de carne magra es aproximadamente $72 \%$ de agua, $21 \%$ de proteínas, $5 \%$ de grasas y $1 \%$ de cenizas (Hui et al., 2006).

La estructura del músculo es fundamental para para entender las relaciones entre las propiedades del músculo y su empleo como carne. El músculo estriado o esquelético, llamado así, debido a que al microscopio se observa una estructura estriada distribuida, a los largo de las fibras musculares, las cuales son la unidad estructural, compuesta de miofibrillas, bastones largos y finos de aproximadamente uno a dos $\mu \mathrm{m}$ de diámetro. Los cortes transversales de las miofibrillas presentan filamentos gruesos y delgados, dando apariencia de bandas claras y oscuras. La banda clara llamada banda I, tiene en su mayoría moléculas de actina mientras la banda obscura llamada banda A, contiene las moléculas de misiona, estas mantienen su ordenamiento trasversal y longitudinal mediante gruesas bandas cruzadas, localizadas periódiet al.camente a lo largo de su longitud, y especialmente por conexiones entre ellos que se alinean en el centro de la banda A. Son estas conexiones las que forman la línea M (Murai et al., 1995). La banda I está dividida por una banda oscura llamada línea Z, la porción de miofibrilla comprendida entre dos líneas $\mathrm{Z}$ adyacentes se llama sarcómero, el cual comprende una banda $\mathrm{A}$ y dos medias bandas I que se localizan a cada lado de la banda A (Vigoreaux, 1994). El sarcómero es la unidad estructural y de contracción de la miofibrilla (Forrest et al., 2001). 
En términos bioquímicos, la carne es el resultado de una serie de transformaciones y reacciones físicas y químicas, que tienen lugar en el músculo después de la muerte del animal durante el proceso de conversión de músculo a carne. Este proceso se lleva a cabo en tres fases: la fase de demora del rigor o pre rigor, comprende el tiempo tras la matanza del animal en que las proteínas del músculo todavía no han sufrido cambios y el músculo aún es elástico; la fase de rigor mortis, consta del acortamiento de los sarcómeros (formación de enlaces entrecruzados entre filamentos finos y gruesos) y la rigidez (tensión continua de la fibras musculares) y la fase de resolución o maduración, la extensibilidad de los músculos se recupera y la carne sufre un proceso de ablandamiento paulatino (Andújar et al., 2009).

En los siguientes apartados se detallarán con mayor profundidad los conceptos básicos que se han mencionado, para una mejor comprensión del lector.

\section{Calidad de la carne}

El concepto de calidad de carne se refiere a las características deseables que el consumidor espera del producto, las preferencias del consumidor están relacionadas con los sentidos humanos, apariencia, olor, sabor y textura al masticar (Sionek \& Przybylski, 2016). Las variables para medir la calidad de la carne fresca son; color, capacidad de retención de agua, perdida por goteo, textura, sabor, contaminación, cantidad de grasa intra, inter y subcutánea, mismas que están relacionadas por factores como; estructura muscular, composición nutricional, factores de manejo ante y post mortem del animal (Joo et al., 2013).

\section{Bioquímica del músculo}

Cuando no hay circulación sanguínea, los músculos ya no pueden obtener energía por medio de la respiración, ya que la actividad mitocondrial cesa por la reducción del oxígeno interno. En consecuencia el glucógeno, la principal reserva de energía del músculo, se convierte en ácido láctico, por la glucólisis post mortem. En comparación con la respiración, la glucólisis post mortem es una forma ineficiente de conseguir energía, el rendimiento de adenosin trifosfato (ATP) en la glucólisis es de 2 a 3 moles de ATP por mol de glucosa, comparado con 38 ATP en la respiración aerobia (Lehninger et al., 2005).

La glucólisis es la ruta primaria en el metabolismo de la glucosa o del glucógeno para sintetizar ATP en condiciones aeróbicas o anaeróbicas, en este proceso se degrada una molécula de glucosa en una serie de reacciones catalizadas enzimáticamente y se producen dos de ácido pirúvico (piruvato en forma de sal). Por esta vía metabólica se obtiene energía en forma de ATP para las células. Este proceso consiste de diez reacciones enzimáticas catabólicas o degradativas en presencia de enzimas endógenas (quinasas, isomerasas, oxidoreductasas, transferasas y aldolasas), que convierten la glucosa en piruvato, sustrato de la respiración celular (Figura 9.1). El piruvato formado en la glucólisis puede seguir varias rutas catabólicas distintas (Figura 9.2). Una de ellas, bajo condiciones anaeróbicas, produce ácido láctico (lactato en forma de sal). El piruvato se metaboliza aeróbicamente en las mitocondrias musculares para lo cual requiere oxígeno, y si se produjera lactato, circula hacia la sangre para transformarse en piruvato.

Durante el proceso, se produce la activación de la ATP-asa no contráctil de la miosina, en lugar de la ATP-asa contráctil de la actomiosina, como consecuencia el sistema enzimático citocromo no puede actuar, imposibilitando la resíntesis del ATP a partir de esta enzima. La acción continua del ATP-asa no contráctil de la miosina, reduce progresivamente el nivel de ATP produciendo simultáneamente fosfato inorgánico, que estimula la degradación del glucógeno a ácido láctico.

La reducida disponibilidad de ATP aumenta la dificultad de mantener la integridad estructural de las proteínas, contribuyendo también a ello la diminución de $\mathrm{pH}$ por la acumulación de ácido láctico. El descenso de $\mathrm{pH}$ hace que las proteínas miofibrilares se aproximen a sus puntos isoeléctricos y frecuentemente la desnaturalización va acompañada de una reducida capacidad de retención de agua (CRA) de dichas proteínas. La conversión de glucógeno a ácido láctico continuará hasta que se alcance un $\mathrm{pH}$ en el que se inactiven las enzimas que efectúan su degradación. 
Figura 9.1 La glucólisis es la vía metabólica que en 10 pasos de reacciones químicas cada uno catalizado por una enzima específica, convierte la glucosa en las células musculares, en 2 moléculas de piruvato, 2 moléculas de adenosin trifosfato (ATP) y 2 moléculas de nicotinamida adenina dinucleótido reducido (NADH). GAPDH=Gliceraldehído 3-fosfato deshidrogenasa. Adaptado de Li et al. (2015)

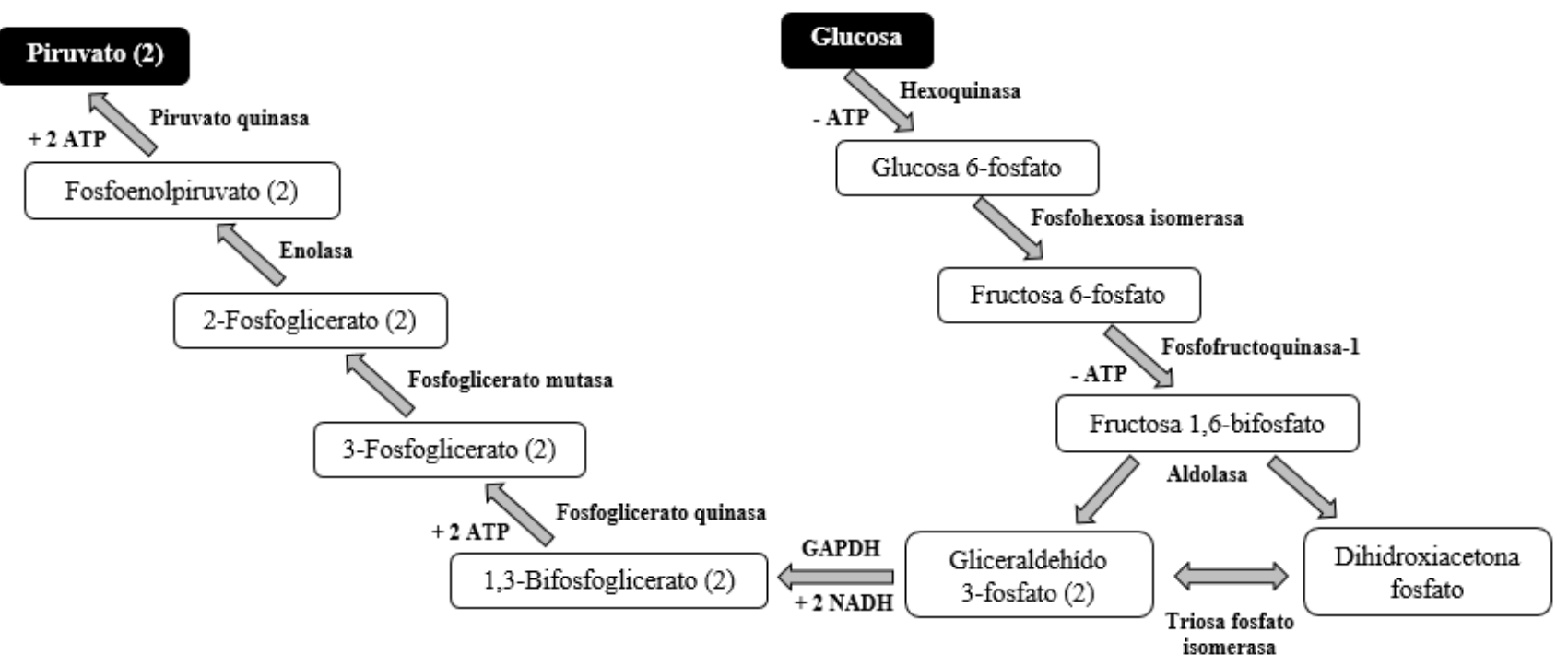

Figura 9.2 Destinos catabólicos del piruvato producido en la glucólisis aerobia y anaerobia. Fuente:

Adaptado de Lehninger et al. (2005)

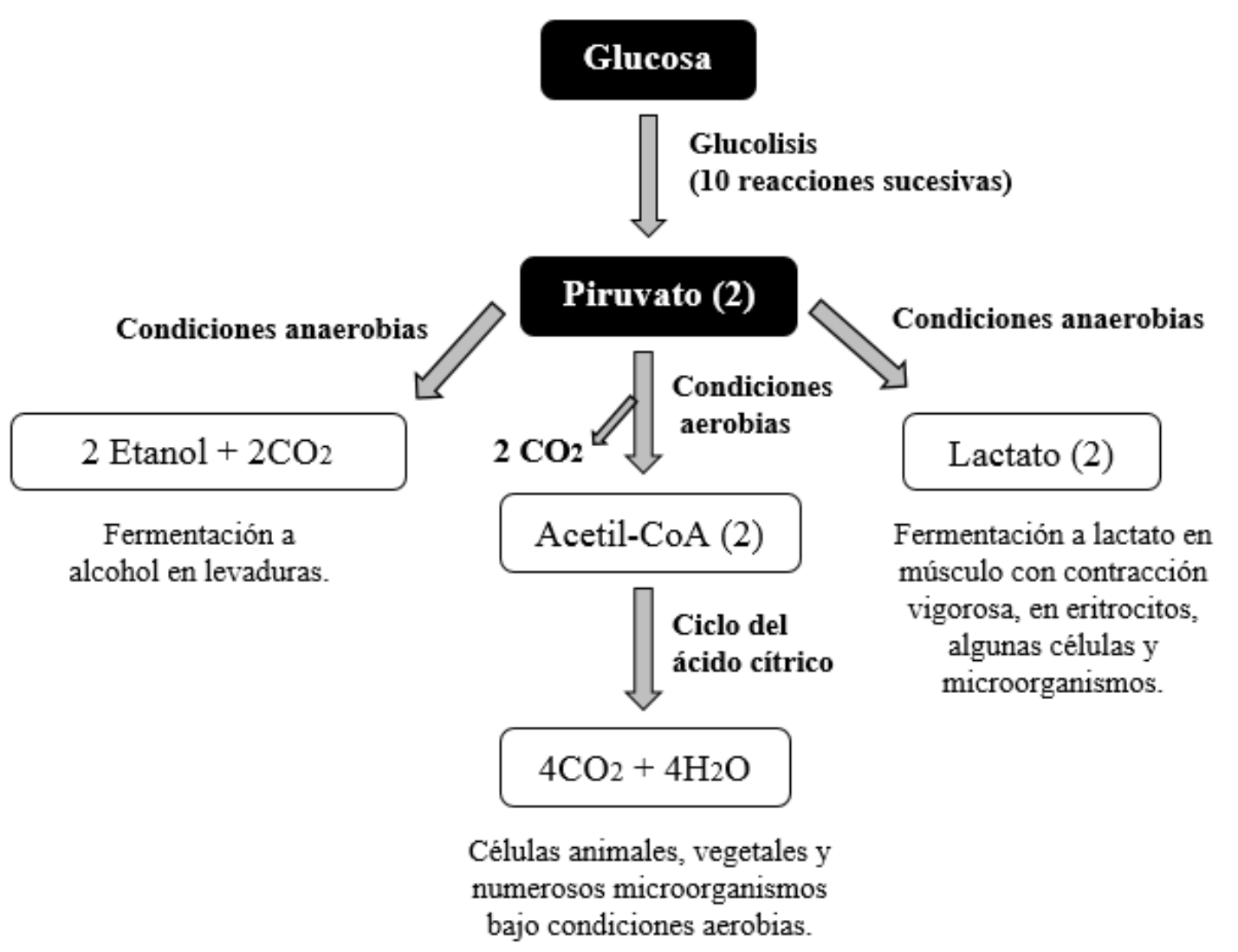

En este sentido, la conversión de los músculos en carne no es un suceso instantáneo, después de ser desangrado el animal, las fibras musculares sobreviven durante algún tiempo mediante glucólisis anaerobia, aunque después de cierto tiempo se agota la energía. Inicialmente se agota su depósito primario de carbohidratos, el glucógeno, y el producto final de la glucólisis anaerobia, el lactato; se perdió en la sangre por lo que no se acidifica la carne. Es entonces cuando las fibras musculares comienzan a perder su integridad al no disponer de energía (Bodwell et al., 1965) y el pH se mantiene cercano a 7. 


\section{Clasificación de la carne}

La clasificación de la carne se relaciona con el proceso de acidificación post mortem. Después de la muerte de animal termina el aporte de oxígeno al músculo, por lo tanto, ya no se puede tener energía por la vía aerobia, y mediante el proceso de glucolisis se obtiene energía por la vía anaerobia, obteniendo de esta forma energía y además ácido láctico, lo que ocasionada que el $\mathrm{pH}$ disminuya. La cinética de descenso pH clasificará la carne en Normal, Pálida Suave y Oxidativa (PSE), y Oscura Firme y Seca (DFD).

\section{PSE}

Esta condición de calidad de la carne se genera por una glucólisis acelerada, desencadenada por un descenso rápido del $\mathrm{pH}$, mientras la temperatura corporal de la canal es aún elevada (Owens \& Sams, 2000). Esto provoca la desnaturalización de aproximadamente el $20 \%$ de las proteínas sarcoplasmáticas y miofibrilares, por consiguiente la disminución de la CRA, mayores pérdidas por goteo y como consecuencia un menor rendimiento por cocción y un textura dura. Esta condición hace a la carne altamente exudativa, le da una apariencia pálida al desnaturalizarse la mioglobina y una textura blanda, poco apetecible para el consumidor.

El valor de $\mathrm{pH}$ promedio a los 45 minutos post mortem es menor a 6, dependiendo de la especie animal y del tipo de musculo, ya que los músculos oxidativos tienen un pH más elevado, respecto a los glucolíticos. Por otra parte la carne PSE se presenta de forma más frecuente en los cerdos, pollos y pavos respecto a la carne de bovino, en el caso de los cerdos el gen del halotano lo hace susceptible a presentar este tipo de carne, cuando los animales son estresados antes de la matanza.

La carne PSE es un problema en la industria de la carne, en el caso de carne de pollo, Barbut (1997) y Woelfel et al., (2002) reportan datos de 30 a $40 \%$ de pollos o pavos afectados por PSE por parvada, afectando la aceptabilidad del consumidor, debido al color pálido, textura poco firme de la carne, baja capacidad de retención de agua y bajo poder de gelificación, lo cual disminuye la calidad y rendimientos de los productos cárnicos elaborados.

\section{DFD}

Esta condición en la carne se genera en animales ejercitados o exhaustos antes de la matanza. Los animales consumen sus reservas de glucógeno, provocando que se alcance una menor concentración de ácido láctico en el proceso de glucólisis post mortem, que conlleva a un $\mathrm{pH}$ final elevado (pH 6.0-6.5). Esta carne tiene una apariencia oscura (la mioglobina se desnaturaliza en menor medida), hay una menor reflexión de la luz, se reduce la difusión del oxígeno hacia el interior y presenta menores pérdidas por goteo que la normal (presenta una alta CRA al estar el pH alejado del punto isoeléctrico de las proteínas musculares), textura firme y gomoso. Esto puede ser producido por tiempos de espera a la matanza excesivamente altos, transportes inadecuados en grandes distancias o ayunos largos, peleas entre animales. Además por el $\mathrm{pH}$ elevado final en carne, aumenta el crecimiento microbiológico, lo que dificulta la conservación de la carne.

\section{Normal}

Este tipo de carne es considerada ideal para consumo como carne fresca o industrialización, ya que se caracteriza por presentar un valor de $\mathrm{pH}$ promedio a los 45 minutos de 6.4 y a las 24 horas post mortem de 5.5 de acuerdo a la especie y tipo de musculo, su apariencia en general es de color de rosa-rojizo, firme, no exudativa y una perdida por goteo menor del goteo del $6 \%$.

\section{$\operatorname{Color}\left(\mathbf{L}^{*}, \mathbf{a}^{*} \mathbf{y} \mathbf{b}^{*}\right)$}

El color se define como la sensación resultante de estimular la retina por las ondas luminosas comprendidas en la región visible del espectro. Los atributos relacionados con el color son el tono, la saturación y la luminosidad. Ésta depende del tipo de músculo y de la concentración de mioglobina que contenga, además del estado de oxidación del átomo de hierro del grupo hemo y de una posible desnaturalización de la globina (Hulot \& Ouhayoun, 1999). El sistema de representación del color más adecuado es el CIELAB (CIE, 1986), ya que produce más uniformidad en la zona de los rojos. 
Este sistema emplea las coordenadas tricromáticas L* (luminosidad), a* (índice color rojo) y b* (índice color amarillo), de manera que a partir de relaciones entre ellas se pueden obtener las coordenadas colorimétricas, la intensidad de color o saturación y el tono. La coordenada $\mathrm{L}^{*}$ es la más relacionada con la valoración visual del consumidor (Murray, 1989). Esta depende de varios factores como el pH, la CRA, la humedad, la integridad de la estructura muscular y, en menor medida, del grado de oxidación de los hemopigmentos (Palombo \& Wijngaards, 1990). La coordenada a* (eje rojo-verde) está relacionada con el contenido de mioglobina, mientras la coordenada $b^{*}$ (eje amarillo-azul) ha sido relacionada con los distintos estados de la mioglobina (Pérez-Álvarez, 1996).El color de la carne es la primera característica que el consumidor va a apreciar y determinante para la decisión de compra.

La mioglobina es la principal responsable de la coloración de la carne, cuya función en el musculo es transportar y almacenar el oxígeno. Está formada por una parte proteica (globina) y una parte no proteica que es un grupo hemo, compuesta por cuatro anillos pirrólicos y un átomo hierro que tiene seis enlaces, uno que está unido a la globina, cuatro unidos a los anillos pirrólicos y un sexto enlace que se puede unir a moléculas como el oxígeno. El color de la carne estará determinado por la cantidad y estado fisicoquímico del pigmento (mioglobina) y estado físico de la carne. La cantidad de pigmento determina la intensidad, la cual varía en función de la especie, edad del animal, tipo de músculo.

El estado químico de la mioglobina (oxido-reducción) determina la tonalidad, asociado al estado de frescura de la carne. En el interior de la carne donde no hay oxigeno se encuentra la desoximioglobina (rojo púrpura), que al tener afinidad con el oxígeno se une a éste y se forma la oximioglobina (rojo brillante), en ambos el hierro se encuentra en forma reducida $\left(\mathrm{Fe}^{2+}\right)$, cuando este se oxida $\left(\mathrm{Fe}^{3+}\right)$ se forma la metamioglobina con un color café, característico de una carne que tiene mucho tiempo en refrigeración o defectuoso de conservación.

La L* estará determinada por el estado físico de la carne (estructura y reflexión de la luz), en el caso de carnes clasificadas como PSE, al bajar el pH rápidamente cuando aún la temperatura de la canal es alta, ocasiona la desnaturalización proteica, provocando una baja capacidad de retención de agua y alta perdida por goteo, esto provoca superficies húmedas, lo que ocasiona una gran reflexión de la luz y una coloración clara, por otra parte, la carne DFD, el pH al bajar de forma leve, lo que ocasiona una elevada retención de agua, provoca superficies secas y una alta absorción de la luz, ocasionado por el empaquetamiento fuerte de la fibras.

El general el color de la carne de pollo es un rosa pálido y ligeramente amarillento dependiendo de los valores que tomen los índices de $\mathrm{L}^{*}, \mathrm{a}^{*} \mathrm{y} \mathrm{b}^{*}$. EL color de la carne de pollo en el musculo pectoralis major se reporta que toma valores de índice de $L^{*}$ de 49.26 a 65.50 , de 5.77 a 16.40 para $b^{*}$ y de -1.23 a 2.53 para $a^{*}$, una carne con valores de índice de $\mathrm{L}^{*}$ superiores a 51 clasifica a la carne como PSE, mientras que valores menores a 48 corresponde a una carne DFD (Bauermeister et al., 2000; Bautista et al., 2016).

\section{pH}

El pH de la carne es determinado por procesos bioquímicos post mortem que ocurren durante la transformación del musculo a carne, que implica cambios continuos en el metabolismo de células musculares, así como la estructura de las proteínas (Andújar et al., 2009). La glucolisis anaerobia es la responsable del descenso del $\mathrm{pH}$ y la fuente de energía de las células del musculo, según la cantidad de glucógeno en este, se producirá el ácido láctico la cual está favorecida por la interrupción de la circulación de la sangre dado el proceso de matanza del animal. Cuando no hay circulación, los músculos ya no pueden obtener energía por medio de la respiración, ya que la actividad mitocondrial cesa por la reducción del oxígeno interno. En consecuencia, el glucógeno, la principal reserva de energía del músculo, se convierte en ácido láctico, por la glucólisis post-mortem.

El pH influye en otras características de la carne, como; el color, textura, capacidad de retención de agua, las cuales determina la calidad y vida de anaquel, ya que existen una gran diversidad de bacterias que se desarrollan en distintos niveles de $\mathrm{pH}$ (Guerrero et al., 2002). El pH en el musculo vivo tiene un valor cercano al 7, después de la muerte del animal desciende según la cantidad de glucógeno que se encuentran en el musculo, que mediante la glucolisis se forma ácido láctico. 
Animales expuestos a un estrés severo en corto tiempo ante mortem, presenta un descenso rápido del pH post mortem entre 5.3 y 5.7 los que es característico de una carne PSE, que se defina con una desnaturalización de proteínas, causando una mayor pérdida de agua y un color rojo pálido, por el contrario animales sometidos a un estrés prolongado o exceso de ayuno ante mortem, el pH será de un 6.4 a 6.8, debido a la poca formación de ácido láctico, que es característico de una carne DFD afectando el color, textura, capacidad de retención de agua y perdida por goteo de la carne. Estos dos tipos de carnes son menos aceptables por los consumidores (Adzitey \& Nurul, 2011; Romero \& Sánchez, 2012).

\section{Perdida por goteo}

El agua es el principal componente de la carne, representa un $85 \%$ aproximadamente, de ella dependen las características como el color, la textura y la jugosidad. El agua en la carne se puede encontrar de tres formas, la primera es el agua ligada que está fuertemente unida al musculo y se mantiene unida al someterla a una fuerza ya sea mecánica o de otro tipo, la segunda es el agua inmovilizada que está ligada más débilmente y que la liberación depende de la cantidad de fuerza física que se ejerce sobre el musculo, la tercera es el agua libre se mantiene únicamente por fuerzas superficiales que es fácilmente desprendible, de esta última es la que tiene mayor importancia ya que influye en el rendimiento de la canal y carne porque se pierde por la evaporación y la perdida por goteo, está perdida es una solución roja acuosa de proteínas que proviene de la superficie del corte muscular en cierto periodo de tiempo y se mide como el exudado del agua extracelular de la carne (Morón-Fuenmayor \& Zamorano-García, 2004).

\section{CRA}

La capacidad de retención de agua es la propiedad de la carne para conservar agua cuando se somete a factores externos como corte o presión, entre otros. Es una propiedad funcional importante de calidad porque influye sobre la carne antes y durante el cocinado. Una alta CRA mantiene la jugosidad de la carne y mejora el rendimiento en el procesamiento (López \& Casp, 2004). La CRA de la carne puede ser afectada por factores como: reducción del pH post mortem, pérdida de ATP, establecimiento del rigor mortis y cambios en la estructura miofibrilar asociados parcialmente con la actividad proteolítica (Young et al., 2004). Por ello, las propiedades físicas más importantes de la carne (color y textura de la carne cocida) están estrechamente relacionadas con la CRA (Hulot \& Ouhayoun, 1999). La CRA determina dos importantes variables económicas: el rendimiento y la calidad de los productos obtenidos. La CRA del tejido muscular tiene efecto directo durante el almacenamiento, cuando los tejidos tienen poca CRA, las pérdidas de humedad y, consecuentemente, la pérdida de peso durante el almacenamiento (mermas) son mayores. Esta pérdida de humedad tiene lugar en las superficies de la canal expuestas a la atmósfera durante el almacenamiento (Forrest et al., 2001). Las pérdidas de peso se producen en toda la cadena de distribución y transformación y pueden ser de 4 a $5 \%$ del peso inicial. Por ello, el estudio de esta propiedad es importante al momento de caracterizar la calidad de la carne (Offer et al., 1989).

Por otro lado, la CRA depende de dos factores fundamentales: el tamaño de la zona $\mathrm{H}$, que es el espacio donde se retiene el agua, y la existencia de moléculas que aporten cargas que permitan establecer enlaces dipolo-dipolo con las moléculas de agua. El agua en la carne está predominantemente escondida en la red de las miofibrillas, incluso tras la homogeneización de la carne. El volumen disponible en la red de miofibrillas es crucial en su capacidad para unir agua. La relativa rigidez de las líneas Z y M impone límites al aumento de volumen. Este aumento también está limitado por las fibras de tejido conectivo y membranas que rodean a la fibra muscular. Un factor limitante del aumento de dicho volumen es la falta de disminución del pH que induce la formación de puentes entre las miofibrillas en el rigor mortis, con la consecuente disminución de la CRA (Wismer-Pedersen, 1994).

\section{Textura}

Hui et al. (2006) definen la textura de la carne como "la dificultad o la facilidad con la que una carne se puede cortar o masticar". Resultante de la resistencia de dos fuerzas: la relacionada con el tejido conectivo y la relacionada con el tejido miofibrilar. La textura depende de un gran número de factores biológicos intrínsecos, tales como: genética del animal, raza, edad, sexo, régimen de alimentación y tipo de músculo, así como la madurez fisiológica del animal al momento de la matanza, factores de manejo de los animales antes de la matanza y de las condiciones post-mortem de la canal y de la carne (Kuber et al., 2004; Hui et al., 2006). 
Según King et al. (2003), las diferencias en textura se deben principalmente a la localización y función del músculo en la vida del animal. Otros estudios mencionan que la madurez fisiológica del animal y la textura están inversamente relacionadas (Boleman et al., 1996; Lawrence et al., 2001). Esto se relaciona con lo encontrado por Kinsman (1994), quien menciona que muchos de los puentes covalentes que unen las moléculas de tropocolágeno son relativamente lábiles en los animales jóvenes y se hacen más estables conforme aumenta la edad. Este descenso en la proporción de puentes covalentes lábiles/estables es el responsable de la contribución del colágeno a la dureza de la carne cocinada (Cross et al., 1973). Por otra parte, Van Hoof (1979), afirma que la textura es influenciada por el tipo de fibras musculares, es decir, el tamaño de los haces de fibras musculares, y el número de fibras que cada uno de ellos contiene, ya que los distintos tipos de estas fibras presentan diferentes capacidades de contracción y de retención de agua y, por tanto, reaccionan de distinta forma a la temperatura; además inciden sobre la dureza, la longitud del sarcómero y de las miofibrillas, de forma que cuanto mayor es el estado de contracción mayor es la dureza. Sin embargo, algunos autores consideran que no existe una relación lineal entre estos dos parámetros (Dunn et al., 1993). Otros afirman que la dureza es completamente independiente de la longitud del sarcómero en los músculos de rápida glucolisis post mortem (Smulders et al., 1990). Davis et al. (1980) afirman que la dureza disminuye a medida que aumenta la longitud del sarcómero.

El consumidor confiere una mayor importancia a la dureza como principal atributo de la textura, siendo uno de los criterios determinantes de la calidad de la carne (Savell et al., 1989; Lawrie, 1998). Es por ello que se han buscado diferentes métodos para evaluar la textura de la carne, la mayoría de los autores utilizan la prueba de corte, como los métodos Allo-Kramer y Warner-Bratzler (Sams et al., 1990; Cavitt et al., 2005).

\section{Rendimiento por cocción}

El rendimiento por cocción es una variable de importancia para la industria cárnica, una alta pérdida de agua influye directamente en la calidad de la carne, además de perjudicar los ingresos obtenidos en el producto final, se estima un rendimiento normal del 75 al $85 \%$ de acuerdo a la especie animal (González et al., 2005).

\section{Rendimiento en canal}

Se define canal cuando el animal ha sido sacrificado y despojado de piel, patas, cabeza y vísceras, y canal fría es cuando han transcurrido 24 horas después del sacrificio y esta ha sido refrigerada (Surak, 1996). Según la norma oficial mexicana NOM-009-ZOO (1994), la carne de las especies animales, autorizadas para consumo humano, es definida como una estructura compuesta por fibras musculares estriadas, acompañada o no de tejido conjuntivo elástico, grasa, fibras nerviosas, vasos linfáticos y sanguíneos. El contenido de grasa, color, humedad, sabor, aroma y textura son atributos que se deben tomar en cuenta para evaluar su calidad, sin adulteraciones y sin residuos tóxicos (Mariezcurrena et al., 2010). La valoración de las canales es importante, en todas las etapas de la cadena comercial de la carne. El detallista tiene que vender cortes de carne con el tamaño, apariencia y composición adecuada, para satisfacer al consumidor, mientras que el mayorista o empacador compra el ganado de los productores, que reúne las características que satisfagan la necesidad del detallista. Por su parte, el ganadero cría y finaliza ganado, que la totalidad de la cadena productiva demande.

\section{Efecto del estrés calórico y tiempo de espera en la calidad de la carne de pollo}

El estrés calórico es uno de los factores ambientales más importantes en la producción y calidad de la carne, ya que ocasionan modificaciones en sus características fisicoquímicas y aceleran su descomposición por la colonización de bacterias patógenas. Bautista et al. (2016) evaluaron el efecto del estrés por calor y tiempo de espera antes de la matanza en el desarrollo de rigor mortis y las características fisicoquímicas de la carne del Pectoralis major en pollo. Se ensayaron 2 tiempos de espera a temperatura ambiente de $24{ }^{\circ} \mathrm{C}$ ( 2 y 8 horas) y 2 horas de espera antes de la matanza a $40{ }^{\circ} \mathrm{C}$. Para el desarrollo del rigor mortis se midió el $\mathrm{pH}$, color $\mathrm{L}^{*}, \mathrm{a}^{*}$ y b* a las $0,1,2,4,8,24,48,96$ y $144 \mathrm{~h}$ en 90 pollos. Para las características fisicoquímicas otros 90 pollos fueron sacrificados y se midió el pH, color, capacidad de retención de agua, pérdida por goteo, resistencia al corte y rendimiento por cocción en carne a las 24 horas post mortem. 
El $\mathrm{pH}$ y color medidos en el tiempo mostraron diferencias entre tratamientos $(\mathrm{p}<0,05)$. Las características fisicoquímicas mostraron diferencias entre tratamiento $(\mathrm{p}<0,05)$ siendo mayor la pérdida por goteo, menor la capacidad de retención de agua y rendimiento por cocción, textura más dura, $\mathrm{pH}$ más bajos e índices de $\mathrm{L}^{*} \mathrm{y} \mathrm{b}^{*}$ altos para la carne de aves de dos horas de espera a temperatura ambiente, lo opuesto sucedió con la carne de aves de dos horas de espera a $40^{\circ} \mathrm{C}$. Se concluye que someter pollos de engorda a condiciones de estrés por calor induce a una carne PSE y un tiempo de espera prolongado induce a una carne DFD. El tiempo de espera a la matanza de $2 \mathrm{~h}$ a temperatura ambiente no afecta negativamente la calidad de la carne.

\section{La carne y su importancia nutricional}

La carne es una fuente nutricional importante en la dieta de la mayoría de las personas. Contiene un alto cantidad de proteínas, incluidos aminoácidos esenciales y otros micronutrientes beneficiosos como el hierro, selenio y vitaminas A, B12 y ácido fólico. Aunque por su alto contenido de ácidos grasos saturados se notifican por un lado efectos adversos del consumo de carne en la enfermedad cardiovascular, obesidad y cáncer; mientras que por su pobre cantidad de carbohidratos se reporta un efecto positivo a las mismas enfermedades (Fernández-Ginés et al., 2005). En múltiples procesos fisiológicos intervienen los componentes de la carne para cubrir nuestras necesidades de proteína, energía y lípidos indispensables para el crecimiento, reproducción y mantenimiento de la salud de las personas. En este contexto, los nutrientes y micronutrientes de la carne participan en el mantenimiento de la homeostasis inmunológica para evitar enfermedades patológicas y otro tipo de trastornos ya sea alimenticios o gastrointestinales. En la Tabla 9.1 podemos apreciar la relación entre nutrientes, micronutrientes y la función inmune (Farhadi \& Ovchinnikov, 2018).

Tabla 9.1 Nutrientes y micronutrientes de la carne importantes en la función inmune

\begin{tabular}{|l|l|}
\hline \multicolumn{2}{|c|}{ Nutriente } \\
$\begin{array}{l}\text { Energía } \\
\text { Lípidos }\end{array}$ & $\begin{array}{l}\text { La desnutrición calórica reduce la inmunidad mediada por células y la respuesta de anticuerpos. La } \\
\text { composición de ácidos grasos de las células inmunes a través de la dieta afecta la fagocitosis, la } \\
\text { señalización en los linfocitos T y la capacidad de presentación de antígenos. }\end{array}$ \\
\hline $\begin{array}{l}\text { Proteínas } \\
\text { /aminoácidos }\end{array}$ & $\begin{array}{l}\text { La proteína es necesaria para la maduración de los órganos del sistema inmune. Se requieren } \\
\text { aminoácidos específicos para una óptima función inmune del tejido linfoide asociado al intestino. }\end{array}$ \\
\hline Zinc & $\begin{array}{l}\text { Es crucial para el desarrollo normal y funcionamiento de las células del sistema inmune (neutrófilos, } \\
\text { células NK, fagocitosis y producción de citocinas). }\end{array}$ \\
\hline Cobre & La deficiencia afecta el sistema inmune innato. \\
\hline Cromo & Reduce el cortisol e incrementa las inmunoglobulinas (IgM). \\
\hline Hierro & La deficiencia compromete el sistema linfoide periférico. \\
\hline Selenio & Catalizan reacciones de óxido-reducción y protegen al hospedero del estrés oxidativo. \\
\hline Vitamina E & $\begin{array}{l}\text { Tiene influencia en la respuesta inmune por neutrófilos mediada por anticuerpos. Estimula a los } \\
\text { linfocitos. }\end{array}$ \\
\hline Vitamina A & $\begin{array}{l}\text { Influye en la celularidad de los órganos linfoides. El ácido retinoico es fundamental para la migración } \\
\text { de linfocitos T y B al intestino. }\end{array}$ \\
\hline Vitamina D & $\begin{array}{l}\text { Tiene efectos inhibitorios sobre la respuesta inmune adquirida y un efecto estimulante sobre la } \\
\text { proliferación de monocitos. }\end{array}$ \\
\hline Vitamina C & $\begin{array}{l}\text { Protege las membranas contra el daño de la peroxidación de lípidos. Alivia de la acción supresora de } \\
\text { los corticoides en los neutrófilos. }\end{array}$ \\
\hline Vitamina B12 & $\begin{array}{l}\text { La carencia de riboflavina tiene efecto negativo en la actividad de los macrófagos. Previene del daño } \\
\text { oxidativo en las células del sistema inmune. Es importante en la inmunidad mediada por células y en la } \\
\text { citotoxicidad. }\end{array}$ \\
\hline
\end{tabular}

\section{La carne como alimento funcional}

Los alimentos funcionales son alimentos que han sido modificados o contienen algún ingrediente que demuestre un efecto positivo en el bienestar del individuo o disminuya los riesgos de enfermedades, más allá de la nutrición básica (Hasler \& Thomson, 2004). Tanto la carne y los productos cárnicos pueden modificarse agregando ingredientes considerados beneficiosos para la salud o eliminando o reduciendo componentes que son considerados dañinos. De esta forma se puede obtener una serie de alimentos que, sin alterar su base, se consideran saludables (Fernández-Ginés et al., 2005). Lo más común es la incorporación de nutracéuticos o productos químicos bioactivos como fibra dietética, oligosacáridos, aminoácidos, glucósidos, vitaminas, minerales, ácidos grasos insaturados, antioxidantes y esteroles vegetales (Brewer, 2012). 
Los beneficios derivados del consumo de alimentos funcionales son reducir el riesgo de desarrollo de enfermedades cardiovasculares, neoplásicas, osteoporosis, arterioesclerosis, hipertensión, obesidad y diabetes.

Las modificaciones de productos cárnicos con fines de promoción de la salud generalmente son para aumentar el contenido de ácidos grasos esenciales, reducir el contenido de sal, reducir el contenido de nitratos, aumentar el contenido de antioxidantes, aumentar el contenido de fibra dietética e incluir probióticos y prebióticos (Spence, 2006; Ostaszewski, 2018).

1. Incrementar los ácidos grasos esenciales. El cambio en la composición y el valor nutricional de los productos cárnicos se obtiene en la etapa de finalización de los animales modificando su dieta mediante la adición de aceite de linaza, algas marinas y aceite de pescado. De esta manera, el contenido de grasa en la canal de cerdo se redujo en aproximadamente un $23 \%$ y en la carne de res en un 6\% (Wood et al., 2008).

2. Reducir el contenido de sal $(\mathrm{NaCl})$. En productos cárnicos es necesario disminuir la sal para reducir los problemas de hipertensión y por lo tanto promover la salud del consumidor. Se ha demostrado que es posible reducir el nivel de sal en los productos cárnicos al 1,7\% sin cambiar sus características sensoriales, lo anterior puede ocurrir debido al uso de otros cloruros $(\mathrm{KCl}$, $\mathrm{MgCl}_{2}, \mathrm{CaCl}_{2}$ ), así como sales sin cloruro como lactatos y fosfatos (Ruusunen \& Puolanne, 2005).

3. Reducir los nitratos. Normalmente se usan para prolongar la vida de anaquel, fijar características organolépticas características (sabor, olor, color), inhibir el crecimiento de bacterias patógenas para evitar la putrefacción de la carne. Se recomienda utilizar un exceso de ácido ascórbico para inhibir eficazmente la formación de N-nitrosaminas en presencia de oxígeno (Drabik-Markiewicz et al, 2009).

4. Aumentar el contenido de antioxidantes. La oxidación de los lípidos es una de las causas del deterioro de la carne y derivados porque su apariencia determina el inicio de una gran cantidad de cambios indeseables en sabor, textura y valor nutricional. Es posible retardar este proceso adicionando antioxidantes en la dieta de los animales y durante los procesos que aumentan el contenido de sustancias bioactivas en el tejido muscular como vitamina E, carotenoides, extractos de plantas como romero, té, uvas, aceitunas y cáscaras de cítricos (Olmedilla-Alonso et al., 2013)

5. Aumentar el contenido de fibra dietética. El aumento de la ingesta de fibra reduce la presión arterial, el colesterol sérico, suprime el apetito, facilita la motilidad intestinal y retarda la absorción de glucosa y lípidos; y por lo tanto, se reducen las probabilidades de desarrollar enfermedades coronarias, accidente cerebrovascular, hipertensión, diabetes, obesidad y algunas enfermedades gastrointestinales. La adición de fibra en la industria cárnica da como resultado una mejor preservación de la forma de los productos cárnicos tratados con calor y un aumento en su eficiencia. La adición de fibra dietética a los productos cárnicos también tiene un efecto positivo en su color. La adición de varios tipos de fibra a los productos cárnicos con un contenido reducido de lípidos reduce su luminosidad $\left(\mathrm{L}^{*}\right)$ y la intensidad del color amarillo $\left(\mathrm{b}^{*}\right)$, al tiempo que aumenta la intensidad del color rojo (a*) (Biswas et al., 2011)

6. Incluir prebióticos y probióticos. Los probióticos son cepas de bacterias (Bifidobacterium lactis, Lactobacillus casei, L. paracasei, L. rhamnosus) que tienen un efecto beneficioso sobre la salud humana como resultado del desarrollo de una microflora intestinal beneficiosa. Los prebióticos son ingredientes alimenticios que estimulan el crecimiento de grupos definidos de microorganismos, mejorando el funcionamiento del cuerpo humano. Los probióticos son capaces de colonizar el sistema digestivo, lo que limita la adhesión de las células patógenas al epitelio intestinal. Los probióticos reducen los niveles de colesterol, mejoran la motilidad intestinal, estimulan la reparación del epitelio intestinal e inhiben el desarrollo de la microflora no deseable (Ostaszewski, 2018). 


\section{Ácido linoleico conjugado}

El ácido linoleico conjugado (ALC) y en particular dos de sus isómeros (cis-9 trans-11 y trans-10, cis12) tienen una elevada función biológica y de forma potencial pueden ayudar a reducir el riesgo de la presencia de algunos tipos de cáncer, en la reducción de grasa corporal y prevención de diabetes (Yang et al., 2015).

Alfaia et al. (2010) indican que el ALC proviene de alimentos de origen animal, y básicamente de rumiantes, cuyos promedios para bovino y ovino, son 2.9 a 4 y $5.6 \mathrm{mg}$ de $\mathrm{ALC} \mathrm{g}^{-1}$ de grasa, respectivamente, comparado con los promedios en cerdo y pollo, los cuales son de 0.6 y $0.9 \mathrm{mg}$ de ALC $\mathrm{g}^{-1}$ de grasa, respectivamente (Chin et al., 1992). Lo anterior coincide con lo reportado por Schmid et al. (2006), con concentraciones de ALC en carne de ovinos y bovinos, en un rango de 4.3 a 19.0 y de 1.2 a $10.0 \mathrm{mg} \mathrm{g}^{-1}$ lípidos, respectivamente, mientras en carne de cerdo, pollo y caballos, el contenido de ALC es menor a $1 \mathrm{mg} \mathrm{g}^{-1}$ de lípidos. Los mismos autores, señalan que la concentración de ALC en carne de ganado bovino proveniente de diferentes países como Argentina, Brasil y EUA, puede variar hasta en un $70 \%$ (3.06 a $6.2 \mathrm{mg} \mathrm{g}^{-1}$ de lípidos), presentando la carne de Argentina y Brasil los valores más elevados de ALC, comparados con los valores de carne proveniente de EUA.

Estas diferencias fueron atribuidas a los distintos sistemas de alimentación entre países, ya que el sistema de alimentación de los animales provenientes de Argentina y Brasil fue prácticamente de tipo extensivo, donde el pastoreo ocupa un papel importante, mientras que los animales provenientes de EUA fueron alimentados con dietas a base de maíz. De la misma manera, Nuernberg et al. (2008) reportaron que corderos en pastoreo muestran mayor concentración del isómero cis-9, trans-11(21.3 mg $100 \mathrm{~g}^{-1} \mathrm{de}$ carne) en músculo graso, en comparación con corderos alimentados con una dieta a base de trigo y avena,

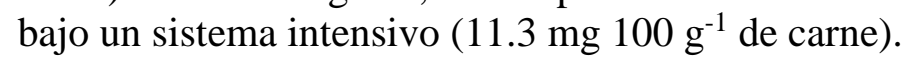

Boles et al. (2005) observaron que la suplementación con aceite de cártamo (6\%) en la dieta de corderos, incrementa el porcentaje de varios isómeros del ALC en músculo magro, sin afectar el crecimiento animal, las características de la canal y la estabilidad del color de la carne. Por su parte, Wachira et al. (2002) observaron que el ALC alcanzó una concentración de 1.65 y 1.90\% en músculo Longissimus dorsi y tejido adiposo subcutáneo de corderos, respectivamente, al suplementar con linaza y aceite de pescado en la dieta. Wynn et al. (2006) evaluaron la efectividad de un suplemento de ALC protegido en las características de la canal y el perfil de ácidos grasos en diferentes tejidos de corderos. Los mismos autores encontraron que las proporciones de los isómeros cis-9, trans-11 y trans-10, cis-12 del ALC, incrementaron en hígado y todos los tejidos estudiados (músculo Longissimus dorsi, hígado, subcutáneo, grasa omental, grasa perirrenal), por efecto de incluir ALC protegido en la dieta. Los resultados de los estudios anteriores demuestran la eficacia de utilizar ALC protegido en la dieta de bovinos y ovinos, donde se ha demostrado que el ALC es depositado en diferentes tejidos, situación que representaría una ventaja para los rumiantes en comparación con no-rumiantes, sobre todo por el potencial efecto anticancerígeno y lipolítico del ALC.

Además de considerar factores relacionados a la dieta y al animal, se deben considerar el proceso de cocción de los alimentos, ya que la temperatura puede modificar la composición de la carne, especialmente la composición de la grasa, debido que los lípidos son rápidamente oxidados por efecto de la temperatura (Badiani et al., 2002), aunque hay gran variabilidad en los cambios relativos individuales de ácidos grasos en respuesta a diferentes métodos de cocción (Badiani et al., 2002). Al respecto, Alfaia et al. (2010) investigaron el efecto de algunas prácticas culinarias como la ebullición, microondas y parrilla, en la composición de ácidos grasos de carne de ganado bovino, con especial énfasis en los isómeros del ALC. Los principales cambios que observaron en la composición de ácidos grasos fueron en 16 ácidos grasos de los 34 estudiados, los cuales resultaron en porcentajes más altos de AGS y AGMS en carne cocida, con menor proporción de ácidos grasos poli-insaturados, en relación con la carne cruda, mientras que los isómeros del ALC mostraron una gran estabilidad a los procesos térmicos. Lo anterior sugiere que los ácidos grasos poliinsaturados son afectados por la temperatura, a excepción del ALC, el cual parece mostrar mayor estabilidad, situación que representa ventaja al ser éste un ácido graso de importancia en la salud humana. 


\section{Microorganismos patógenos en la carne}

Durante el sacrificio y el procesamiento de los animales la superficie muscular puede contaminarse con microorganismos procedentes de la piel, heces y el contenido intestinal. Siendo el tejido muscular una excelente plataforma para el crecimiento bacteriano ya que tiene un $\mathrm{pH}$ neutro, alto contenido de humedad, proteínas y lípidos, todo lo necesario para la supervivencia de las bacterias. La carne y los productos cárnicos son fuentes importantes de infecciones humanas por transmitir bacterias en los alimentos. Los principales géneros de bacterias presentes en las superficies musculares son Pseudomonas spp., Acinetobacter spp., Aeromonas spp., Brochothrix thermosphacta, bacterias del ácido lácticas como Lactobacillus, así como muchos miembros de Enterobacteriaceae incluyendo Enterobacter y Serratia spp. (Huis, 1996). Sin embargo, la presencia de patógenos en la carne como Salmonella spp., Campylobacter jejuni, Escherichia coli verotoxigénica y Listeria monocytogenes, ha ocasionado desde hace mucho tiempo severos brotes de enfermedades alimentarias. Estos patógenos deben controlarse mediante un sistema continuo de inspección sanitaria mediante acciones y métodos que permitan la detección eficaz desde la matanza, procesamiento, almacenamiento, distribución y comercialización de la carne, incluyendo el manejo que se da en el hogar para evitar más contaminación cruzada.

1. Salmonella. La Salmonella spp. ha sido reconocida como los principales patógenos transmitidos por los alimentos, causan gastroenteritis, fiebre entérica y bacteriemia. Las serovariedades más comunes que causan enfermedades humanas y animales son Salmonella typhi, Salmonella paratyphi, Salmonella enteriditis, Salmonella typhimurium y Salmonella choleraesuis. Las especies de Salmonella son gramnegativas, generalmente móviles, facultativas, anaerobias, no formadoras de esporas y tienen forma de bastón. Las enfermedades causadas por estas bacterias se denominan salmonelosis, fiebre tifoidea y fiebre paratifoidea que son dos formas graves de salmonelosis. En el mundo siguen siendo importantes debido a la pobreza, la aparición de múltiples cepas resistentes a los medicamentos y la falta antibióticos y vacunas sobre todo en países en desarrollo. Numerosos serotipos son capaces de causar infección en aves de corral donde colonizan el tracto digestivo en aves jóvenes, aunque hay cepas que pueden invadir órganos internos, como bazo, hígado y tracto reproductivo, siendo estas cepas las más difíciles de controlar para la industria avícola (Hu et al., 2003).

2. Campylobacter. Campylobacter spp. Son patógenos zoonóticos y los principales causantes de gastroenteritis transmitida por alimentos en humanos en todo el mundo. Campylobacter también puede desencadenar serias secuelas post-infecciosa como la artritis reactiva y el síndrome de Guillain-Barré. Las especies más importantes son Campylobacter jejuni y Campylobacter coli. Las especies de Campylobacter son pequeñas, microaerófilas, espirales y de forma redondeada, gramnegativas con un flagelo sin funda en uno o ambos extremos. La principal ruta de transmisión de Campylobacter es por la ingesta de alimentos o agua contaminados, la infección en los animales domésticos es muy común, siendo las aves de corral, cerdos, ovejas y vacas los más afectados. La población humana se infecta a través del consumo de carne poco cocida de los animales mencionados. Los síntomas comunes de la campilobacteriosis humana incluyen diarrea, fiebre, calambres abdominales, malestar general y dolor de cabeza. Aproximadamente el $10 \%$ de los casos confirmados por laboratorio requieren hospitalización (Altekruse et al., 1999)

3. Escherichia coli. Pertenece a la familia Enterobacteriacea, es catalasa positive, oxidasa negativa, gran negativa, sin esporas, es un residente normal del intestino de animales de sangre caliente incluido el humano. Estos pueden contaminar productos alimenticios durante el sacrificio de animales y evisceración o durante la manipulación de alimentos. Naturalmente, se encuentra en ambientes como el agua, suelo y heces. La presencia de este organismo indica malas prácticas de higiene o procesamiento inadecuado. Los síntomas de infección incluyen fiebre, vómitos, estómago, calambres y diarrea. E. coli O157: H7 es una cepa de reciente reconocimiento que causa colitis hemorrágica y síndrome hemolítico urémico en humanos, que puede provocar insuficiencia renal aguda. Este microorganismo produce la toxina Shiga en el ganado bovino, sin embargo, también se ha aislado de otros animales, como cabras, ovejas, caballos, perros, cerdos y moscas. E. coli O157: H7 ha sido una preocupación importante en la industria cárnica por décadas, esta bacteria puede hallarse en todas las fases del proceso de sacrificio, se aísla frecuentemente de los cuchillos y sierras que se han convertido en vectores para transferir la bacteria a otros cortes de carne dando lugar a contaminaciones masivas de carne sobre todo la molida. 
Las medidas preventivas para las infecciones por E. coli O157: H7 son similares a las recomendadas para otras enfermedades transmitidas por alimentos como la higiene básica de los alimentos, aplicar los programas estandarizados de sanitización, análisis de riesgos e inspección de los puntos críticos de control de todo el proceso de matanza y elaboración de productos cárnicos (Heuvelink et al., 1996).

4. Listeria. Listeria monocytogenes es una bacteria intracelular capaz de crecer dentro de monocitos y neutrófilos, gran positiva, catalasa positiva, oxidasa negativa, hemolítico, móvil a $20^{\circ} \mathrm{C}-$ $28^{\circ} \mathrm{C}$ con uno a cinco flagelos peritico. L. monocytogenes puede adaptarse para sobrevivir y crecer en condiciones ambientales adversas como como bajas temperaturas, altas concentraciones de sal y $\mathrm{pH}$ ácido, es capaz de sobrevivir en temperaturas de -7 a $45^{\circ} \mathrm{C}$ por lo que puede multiplicarse en alimentos refrigerados. En 2006, se reportó que era la quinta infección zoonótica más común en el mundo. Raramente se encuentra en aves vivas, pero llega a la carne como resultado de la contaminación cruzada durante el procesamiento. Se ha notificado que el consumo de carne cruda o insuficientemente cocida de pollo, res, cerdo y pescado es la principal manera infectarse y desarrollar la listeriosis con mortalidad del 20-30\%, provocando a las personas meningitis, septicemia y aborto (Allerberger \& Wagner, 2010)

\section{Conclusión}

La ciencia y tecnología de la carne es un pilar en el desarrollo de nuestro país, por lo que es imprescindible conocer su composición, sus atributos nutricionales y su cuidado durante su procesamiento. Sin duda, estos conocimientos traerán beneficios para la salud de los consumidores y potenciará la economía de este sector de la industria.

\section{Referencias}

Adzitey, F., \& Nurul, H. (2011). Pale soft exudative (PSE) and dark firm dry (DFD) meats: Causes and measures to reduce these incidences - a mini review. International Food Research Journal, 18(1), 1120.

Alfaia, C.M., Alves, S.P., Lopes, A. F., Fernandes, M.J., Costa, A. S., Fontes, C.M., Castro, M.L.F., Bessa, R.J.B., \& Prates, J.A. (2010). Effect of cooking methods on fatty acids, conjugated isomers of linoleic acid and nutritional quality of beef intramuscular fat. Meat Science, 84(4): 769-777.

Allerberger, F., \& Wagner, M. (2010). Listeriosis: a resurgent foodborne infection. Clinical Microbiology and Infection, 16(1), 16-23.

Altekruse, S.F., Stern, N.J., Fields, P.I., \& Swerdlow, D.L. (1999). Campylobacter jejuni -an emerging foodborne pathogen. Emerging infectious diseases, 5(1), 28.

Andújar, G., Pérez, D., \& Venegas, O. (2009). Los cambios post mortem y la trasformación del músculo en carne. En: Química y bioquímica de la carne y productos cárnicos. Instituto de Investigaciones para la industria alimentaria. Editorial Universitaria. La Habana, Cuba. 125 p.

Badiani, A., Stipa, S., Bitossi, F., Gatta, P. P., Vignola, G., \& Chizzolini, R. (2002). Lipid composition, retention and oxidation in fresh and completely trimmed beef muscles as affected by common culinary practices. Meat Science, 60(2): 169-186.

Barbut, S. (1997). Problem of pale soft exudative meat in broiler chickens. British Poultry Science, 38(4), 355-358.

Bauermeister L.J., Lewis S.J., \& McKee S.R. (2000). Using microbial transglutaminase to improve meat binding characteristics of PSE poultry meat. Poultry Sci., 2000, 79 (Suppl.1), Abstract 232, 53.

Bautista, Y., Narciso, C., Pro, A., Hernández, A.S., Becerril, C.M., Sosa, E., \& Velasco, J. (2016). Efecto del estrés por calor y tiempo de espera ante mortem en las características fisicoquímicas y la calidad de la carne de pollo. Archivos de Medicina Veterinaria, 48(1), 89-97. 
Biswas, A. K., Kumar, V., Bhosle, S., Sahoo, J., \& Chatli, M. K. (2011). Dietary fibers as functional ingredients in meat products and their role in human health. International Journal of Livestock Production, 2(4), 45-54.

Bodwell, C.E., Pearson, A.M., \& Spooner, M. E. (1965). Post-mortem changes in muscle. I. Chemical changes in beef. Journal of Food Science, 30(5), 766-772.

Boleman, S.J., Miller, R.K., Buyck, M.J., Cross, H.R., \& Savell, J.W. (1996). Influence of realimentation of mature cows on maturity, color, collagen solubility, and sensory characteristics. Journal of Animal Science, 74(9), 2187-2194.

Boles, J.A., Kott, R.W., Hatfield, P.G., Bergman, J.W., \& Flynn, C.R. (2005). Supplemental safflower oil affects the fatty acid profile, including conjugated linoleic acid, of lamb. Journal of Animal Science, 83(9): 2175-2181.

Brewer, M.S. (2012). Reducing the fat content in ground beef without sacrificing quality: A review. Meat Science, 91(4), 385-395.

Cavitt, L.C., Meullenet, J.F., Gandhapuneni, R.K., Youm, G.W., \& Owens, C.M. (2005). Rigor development and meat quality of large and small broilers and the use of Allo-Kramer shear, needle puncture, and razor blade shear to measure texture. Poultry science, 84(1), 113-118.

Chin, S.F., Liu, W., Storkson, J.M., Ha, Y.L., \& Pariza, M.W. (1992). Dietary sources of conjugated dienoic isomers of linoleic acid, a newly recognized class of anticarcinogens. Journal of Food Composition and Analysis, 5(3): 185-197.

CIE (Commission International de l'Eclairage) (1986). Colorimetry, 2da Ed. Viena. 82 p.

Cross, H.R., Carpenter, Z.L., \& Smith, G.C. (1973). Effects of intramuscular collagen and elastin on bovine muscle tenderness. Journal of Food Science, 38(6), 998-1003.

Davis, G.W., Dutson, T.R., Smith, G.C., \& Carpenter, Z.L. (1980). Fragmentation procedure for bovine longissimus muscle as an index of cooked steak tenderness. Journal of Food Science, 45(4), 880-884.

Drabik-Markiewicz, G., Van den Maagdenberg, K., De Mey, E., Deprez, S., Kowalska, T., \& Paelinck, H. (2009). Role of proline and hydroxyproline in N-nitrosamine formation during heating in cured meat. Meat Science, 81(3), 479-486.

Dunn, A.A., Kilpatrick, D.J., \& Gault, N.F.S. (1993). Influence of ultimate pH, sarcomere length and cooking loss on the textural variability of cooked M. pectoralis major from free range and standard broilers. British Poultry Science, 34(4), 663-675.

Farhadi, S., \& Ovchinnikov, R.S. (2018). The relationship between nutrition and infectious diseases: A review. Biomedical and Biotechnology Research Journal (BBRJ), 2(3), 168.

Fernández-Ginés, J.M., Fernández-López, J., Sayas-Barberá, E., \& Pérez-Alvarez, J. A. (2005). Meat products as functional foods: A review. Journal of Food Science, 70(2), R37-R43.

Forrest, J.C., Aberle, E.D., Hedrich, H.D., Hudge, M.D., \& Merkel, R.A. (2001). Fundamentos de la ciencia de la carne. 2da Ed. Zaragoza, España. 365 p.

González, F.A.N., Macías, J.A.G., Bautista, J.H., \& Castro, J.A.J. (2005). Caracterización de canales de ganado bovino en los valles centrales de Oaxaca. Revista Mexicana de Ciencias Pecuarias, 43(2), 219228.

Guerrero, L.I., Ponce, A.E., \& Pérez, M.L. (2002). Curso práctico de tecnología de carnes y pescado. Universidad Metropolitana Unidad Iztapalapa. D. F., México. 171 p. 
Hasler, C. M., Bloch, A. S., \& Thomson, C. (2004). Position of the American Dietetic Association: functional foods. Journal of the American Dietetic Association, 104(5), 814-826.

Heuvelink, A.E., Wernars, K., \& de Boer, E.N.N.E. (1996). Occurrence of Escherichia coli O157 and other verocytotoxin-producing E. coli in retail raw meats in the Netherlands. Journal of food protection, 59(12), 1267-1272.

Hu, L., \& Kopecko, D.J. (2003). Salmonella. In International Handbook of Foodborne Pathogens; Miliotis M. D., Bier, J., Eds.; Marcel Dekker Inc.: New York, NY; Chapter 10, pp 151-67.

Hui, Y., Guerrero, H.I., \& Rosmini, M. (2006). Ciencia y Tecnología de Carnes. Editorial Limusa, S.A. de C.V. Grupo Noriega Editores. Balderas 95, Mexico, D.F.

Huis in 't Veld JH. (1996). Microbial and biochemical spoilage of foods: an overview. International journal of food microbiology, 33(1), 1-18.

Hulot, F., \& Ouhayoun, J. (1999). Muscular pH and related traits in rabbits: a review. World Rabbit Science, $7(1)$.

Joo, S.T., Kim, G.D., Hwang, Y.H., \& Ryu, Y.C. (2013). Control of fresh meat quality through manipulation of muscle fiber characteristics. Meat science, 95(4), 828-836.

King, D.A., Dikeman, M.E., Wheeler, T.L., Kastner, C.L., \& Koohmaraie, M. (2003). Chilling and cooking rate effects on some myofibrillar determinants of tenderness of beef. Journal of Animal science, 81(6), 1473-1481.

Kinsman, D.M., Kotula, A.W., \& Breidenstein, B.C. (1994). Muscle Foods. Meat Poultry and Seafood

Kuber, P.S., Busboom, J.R., Huff-Lonergan, E., Duckett, S.K., Mir, P.S., Mir, Z., McCormick, R.J., Dodson, M.V., Gaskins, C.T., Cronrath, J.D., Marks, J.D. \& Reeeves, J.J. (2004). Effects of biological type and dietary fat treatment on factors associated with tenderness: I. Measurements on beef longissimus muscle. Journal of Animal Science, 82(3), 770-778.

Lawrence, T.E., Whatley, J.D., Montgomery, T.H., Perino, L.J., \& Dikeman, M.E. (2001). Influence of dental carcass maturity classification on carcass traits and tenderness of longissimus steaks from commercially fed cattle. Journal of Animal Science, 79(8), 2092-2096.

Lawrie, R.A. (1998). Ciencia de la carne. 4a Ed. Zaragoza, España. 425

Lehninger, A.L., Nelson, D.L., \& Cox, M.M. (2005). Princípios de bioquímica. Ediciones Omega.

Li, X. B., Gu, J. D., \& Zhou, Q. H. (2015). Review of aerobic glycolysis and its key enzymes-new targets for lung cancer therapy. Thoracic cancer, 6(1), 17-24.

López, R.V., \& A.V. Casp. (2004). Tecnología de los mataderos. 1ra Ed. México, D. F. 430 p.

Mariezcurrena Berasain, M.A., Braña Varela, D., Partida de la Peña, J.A., Ramírez Rodríguez, E., \& Domínguez Vara, I. (2010). Estandarización de la metodología para la determinación de grasa en la carne de cerdo. Revista Mexicana de Ciencias Pecuarias, 1(3), 269-275.

Morón-Fuenmayor, O., \& Zamorano-García, L. (2004). Pérdida por goteo en carne cruda de diferentes tipos de animales. Revista Científica, 14 (1).

Murai, S., Arata, T., \& Inoue, A. (1995). Binding of myosin and its subfragment-1 with antibodies specific to the two heads of the myosin molecule. The Journal of Biochemistry, 117(5), 974-979.

Murray, A.C. (1989). Factors affecting beef color at time of grading. Canadian Journal of Animal Science, 69(2), 347-355. 
NOM-009-ZOO. (1994) Proceso sanitario de la carne. Secretaría de Agricultura, Ganadería, Desarrollo Rural, Pesca y Alimentación. Diario Oficial de la Federación, 31(07).

Nuernberg, K., Fischer, A., Nuernberg, G., Ender, K., \& Dannenberger, D. (2008). Meat quality and fatty acid composition of lipids in muscle and fatty tissue of Skudde lambs fed grass versus concentrate. Small Ruminant Research, 74(1): 279-283.

Offer, G., Knight, P., Jeacocke, R., Almond, R., Cousins, T., Elsey, J., \& Purslow, P. (1989). The structural basis of the water-holding, appearance and toughness of meat and meat products. Food Structure, 8(1), 17.

Olmedilla-Alonso, B., Jiménez-Colmenero, F., \& Sánchez-Muniz, F.J. (2013). Development and assessment of healthy properties of meat and meat products designed as functional foods. Meat Science, 95(4), 919-930.

Ostaszewski, M. (2018). Meat and meat products as functional food. World Scientific News, 110, 147158.

Owens, C.M., \& Sams, A.R. (2000). The influence of transportation on turkey meat quality. Poultry Science, 79(8), 1204-1207.

Palombo, R., \& Wijngaards, G. (1990). Characterization of changes in psychometric colour attributes of comminuted porcine lean meat during processing. Meat Science, 28(1), 61-76.

Pérez-Álvarez, J.A. (1996). Contribución al estudio objetivo del color en productos cárnicos crudocurados. Tesis Doctoral. Universidad Politécnica de Valencia, Valencia. 276 p.

Romero, M.P., \& Sánchez, J.V. (2012). Bienestar animal durante el transporte y su relación con la calidad de la carne bovina. Revista MVZ Córdoba, 17(1), 2936-2944.

Ruusunen, M., \& Puolanne, E. (2005). Reducing sodium intake from meat products. Meat Science, 70(3), 531-541.

Sams, A.R. (1990). Electrical stimulation and high temperature conditioning of broiler carcasses. Poultry Science, 69(10), 1781-1786.

Savell, J. W., Cross, H.R., Francis, J.J., Wise, J.W., Hale, D.S., Wilkes, D.L., \& Smith, G.C. (1989). National consumer retail beef study: Interaction of trim level, price and grade on consumer acceptance of beef steaks and roasts. Journal of Food Quality, 12(4), 251-274.

Schmid, A., Collomb, M., Sieber, R., \& Bee, G. (2006). Conjugated linoleic acid in meat and meat products: A review. Meat Science, 73(1): 29-41.

Sionek, B., \& Przybylski, W. (2016). The Impact of Ante-and Post-Mortem Factors on the Incidence of Pork Defective Meat-A Review. Annals of Animal Science, 16(2), 333-345.

Smulders, F.J.M., Marsh, B.B., Swartz, D.R., Russell, R.L., \& Hoenecke, M.E. (1990). Beef tenderness and sarcomere length. Meat Science, 28(4), 349-363.

Spence, J.T. (2006). Challenges related to the composition of functional foods. Journal of Food Composition and Analysis, 19, S4-S6.

Surak, J. (1996). Un solo sistema no es suficiente para alcanzar la calidad total. Carnetec, 5, 24-27.

Van Hoof, J. (1979). Influence of ante-and peri-mortem factors on biochemical and physical characteristics of turkey breast muscle. Veterinary Quarterly, 1(1), 29-36.

Vigoreaux, J.O. (1994). The muscle Z band: lessons in stress management. Journal of Muscle Research \& Cell Motility, 15(3), 237-255. 
Wachira, A.M., Sinclair, L.A., Wilkinson, R.G., Enser, M., Wood, J.D., \& Fisher, A.V. (2002). Effects of dietary fat source and breed on the carcass composition, n-3 polyunsaturated fatty acid and conjugated linoleic acid content of sheep meat and adipose tissue. British Journal of Nutrition, 88(06): 697-709.

Wismer-Pedersen, J. (1994). Ciencia de la carne y de los productos cárnicos. 3ra Ed. Zaragoza. España. $581 \mathrm{p}$.

Woelfel, R.L., Owens, C.M., Hirschler, E.M., Martinez-Dawson, R., \& Sams, A.R. (2002). The characterization and incidence of pale, soft, and exudative broiler meat in a commercial processing plant. Poultry Science, 81(4), 579-584.

Wood, J.D., Enser, M., Fisher, A.V., Nute, G.R., Sheard, P.R., Richardson, R.I., ... \& Whittington, F.M. (2008). Fat deposition, fatty acid composition and meat quality: A review. Meat Science, 78(4), 343-358.

Wynn, R.J., Daniel, Z.C.T.R., Flux, C.L., Craigon, J., Salter, A.M., \& Buttery, P.J. (2006). Effect of feeding rumen-protected conjugated linoleic acid on carcass characteristics and fatty acid composition of sheep tissues. Journal of Animal Science, 84(12): 3440-3450.

Yang B., Chen H., Stanton C., Ross R.P., Zhang H., Chen Y. Q., \& Chen W. (2015). Review of the roles of conjugated linoleic acid in health and disease. Journal Functional Foods. 15: 314-325.

Young, J.F., Karlsson, A.H., \& Henckel, P. (2004). Water-holding capacity in chicken breast muscle is enhanced by pyruvate and reduced by creatine supplements. Poultry Science, 83(3), 400-405. 